\title{
NEW CONDITIONS ON EXISTENCE AND GLOBAL ASYMPTOTIC STABILITY OF PERIODIC SOLUTIONS FOR BAM NEURAL NETWORKS WITH TIME-VARYING DELAYS
}

\author{
Zhengqiu Zhang and Zheng Zhou
}

\begin{abstract}
In this paper, the problem on periodic solutions of the bidirectional associative memory neural networks with both periodic coefficients and periodic time-varying delays is discussed. By using degree theory, inequality technique and Lyapunov functional, we establish the existence, uniqueness, and global asymptotic stability of a periodic solution. The obtained results of stability are less restrictive than previously known criteria, and the hypotheses for the boundedness and monotonicity on the activation functions are removed.
\end{abstract}

\section{Introduction}

A class of two-layer interassociative network called bidirectional associative memory (BAM) neural network is an important model with the ability of information association, which is crucial for application in pattern recognition, solving optimization problems and automatic control engineering [16, 17]. In such applications, the dynamical characteristics of networks play an important role. As is well known, in both biological and man-made neural networks, time delays occur due to finite switching speed of the amplifiers and communication time. The delays are usually time-varying, and sometimes vary violently with time. They slow down the transmission rate and can influence the stability of designed neural networks by creating oscillatory or unstable phenomena [3]. So it is more in accordance with this fact to study the BAM neural networks with time-varying delays. The circuits diagram and connection pattern implementing for the delayed BAM neural networks can be found in [8]. In recent years, some useful results on the uniqueness and global stability of the equilibrium point for the delayed recurrent neural networks and delayed BAM neural

Received July 1, 2009; Revised March 15, 2010.

2010 Mathematics Subject Classification. 34K13, 34K20, 34K25.

Key words and phrases. periodic solution, BAM neural networks, global asymptotic stability, coincidence degree theory, Lyapunov functional.

Project supported by Doctoral Fund of Ministry of Education of China (No: 200805321017), Postdoctoral fund of China (No: 20060400267), the Scientific Research Foundation for the Returned Oversears Chinese Scholars, State Education Ministry. 
networks have been given, for example, see [1, 2, 3], [8], [13], [15], [17, 18, 19] and references therein. It is well known that studies on neural dynamical system not only involve a discussion of stability properties, but also involve many dynamic behavior such as periodic oscillatory behavior, synchronization, dissipativity, bifurcation, and chaos $[5,8,9]$. In [9], the authors studied the global point dissipativity of neural networks with mixed time-varying delays. In [5], based on the invariant principle of functional differential equations, a simple adaptive feedback scheme is proposed for the synchronization of almost all kinds of coupled identical neural networks with or without time-varying delays. In many applications, the properties of periodic oscillatory solutions are of great interest, it has been found applications in learning theory, which is motivated by the fact that learning usually requires repetition. Hence, it is of prime importance to study periodic oscillatory solutions of neural networks. In addition, an equilibrium point can be viewed as a special periodic solution of neural networks with arbitrary periodic. In this sense, the analysis of periodic solutions of neural networks may be considered to be more general than that of equilibrium point. Recently, periodic solution for BAM neural networks with delays has been studied, for example, see $[4,6,7,8,10,11,12,20,21]$ and references therein. In $[5,7,8,10,12]$, some sufficient conditions ensuring the existence, uniqueness and global exponential stability of periodic solution were given for BAM neural networks with constants delays. In [4, 20, 21], under the hypothesis for the boundedness or monotonicity on the activation functions and the differentiability on the time-varying delays, the authors gave several sufficient conditions ensuring the existence and global exponential stability of periodic solution for BAM neural networks with time-varying delays. However, in some applications, one requires to use unbounded activation functions. For example, when neural networks are designed for solving optimization problems in the presence of constrains (linear, quadratic, or more general programming problems), unbounded activations modeled by diode-like exponential-type functions are needed to impose constraints satisfaction. The extension of the quoted results to the unbounded case is not straightforward. When considering the widely employed piecewise-linear neural networks, infinite intervals with zero slope are present in activations, it is of great interest to drop the assumptions of monotonicity. Therefore, it seems that for some purposes, nonmonotonic functions might be better candidates for neural activation in designing and implementing an artificial neural networks. Motivated by the above discussion, in [22], the authors were concerned with the following model:

$$
\begin{aligned}
\frac{d u_{i}(t)}{d t}= & -a_{i}(t) u_{i}(t) \\
& +\sum_{j=1}^{m} h_{i j}(t) f_{j}\left(\lambda_{j} v_{j}\left(t-\tau_{i j}(t)\right)+I_{i}(t), i=1,2, \ldots, n\right.
\end{aligned}
$$




$$
\begin{aligned}
\frac{d v_{j}(t)}{d t}= & -b_{j}(t) v_{j}(t) \\
& +\sum_{i=1}^{n} w_{j i}(t) g_{i}\left(\mu_{i} u_{i}\left(t-\sigma_{j i}(t)\right)+J_{j}(t), j=1,2, \ldots, m,\right.
\end{aligned}
$$

for $t>0$, with initial value conditions

$$
\left\{\begin{array}{l}
u_{i}(s)=\phi_{i}(s), i=1,2, \ldots, n, s \in[-\sigma, 0], \sigma=\max _{1 \leq i \leq n, 1 \leq j \leq m, 0 \leq t \leq \omega}\left\{\sigma_{j i}(t)\right\} \\
v_{j}(s)=\psi_{j}(s), j=1,2, \ldots, m, s \in[-\tau, 0], \tau=\max _{1 \leq i \leq n, 1 \leq j \leq m, 0 \leq t \leq \omega}\left\{\tau_{i j}(t)\right\} .
\end{array}\right.
$$

in which, $u(t)=\left(u_{1}(t), u_{2}(t), \ldots, u_{n}(t)\right)^{T} \in \mathbb{R}^{n}, v(t)=\left(v_{1}(t), v_{2}(t), \ldots, v_{m}(t)\right)^{T}$ $\in \mathbb{R}^{m}, u_{i}(t)$ and $v_{j}(t)$ are the state of the $i$ th neurons from the neural field $F_{U}$ and the $j$ th neurons from the neural field $F_{V}$ at time $t$, respectively; $f_{j}, g_{i}$ denote the activation functions of the $j$ th neurons from $F_{V}$ and the $i$ th neurons from $F_{U}$ at time $t$, respectively; $I_{i}(t)$ and $J_{j}(t)$ denote the external inputs on the $i$ th neurons from $F_{U}$ and the $j$ th neurons from $F_{V}$, respectively; $\tau_{i j}(t)$ and $\sigma_{j i}(t)$ correspond to the transmission delays and $\tau_{i j}(t)>0$ and $\sigma_{j i}(t)>0 ; a_{i}(t)>0$ and $b_{j}(t)>0$ represent the rate with which the $i$ th neuron from $F_{U}$ and the $j$ th neurons from $F_{V}$ will reset their potential to the resting state in isolation when disconnected from the networks and external inputs, respectively; $h_{i j}(t)$ and $w_{j i}(t)$ denote the connection strengths; $\lambda_{j}>0$ and $\mu_{i}>0$ are constants, correspond to the neural gains associated with the neural activations $[3,13]$.

In [22], the authors gave some novel sufficient conditions ensuring the existence, uniqueness, and global exponential stability of periodic solution for the system (1.1) with both periodic coefficients and periodic time-varying delays by using analytic methods, inequality technique and $M$-matrix theory.

However, to the best of our knowledge, the results on asymptotic stability of periodic solution of the system (1.1) are scarce. So, in this paper, the objective of us is to establish new sufficient conditions ensuring the existence, uniqueness, and global asymptotic stability of periodic solution for the system (1.1) with both periodic coefficients and periodic time-varying delays by using coincidence degree theory, inequality technique and Lyapunov functional. Our results on the existence, uniqueness, and global stability of periodic solution for the system (1.1) with both periodic coefficients and periodic time-varying delays not only remove the hypotheses as required in $[5,20,21]$ for boundedness and monotonicity on the activation functions and remove the restriction that the value of activation functions at zero point is zero in $[12,10]$, but also are more concise than those obtained in $[4,6,7,8,10,12,20,21,22]$ and more easy to verified. In our results of stability of periodic solution, we only need the activation functions satisfy Lipschitz condition and the parameters satisfy two very simple inequalities, while in [22], the matrix of parameters is required to be a complicated $M$ matrix, while in [12], one hand, the matrix of parameters is required to be a complicated $M$ matrix, on the other hand, the parameters are 
needed to satisfy four complicated inequalities, while in [10], the parameters are needed to satisfy four very complicated inequalities.

The paper is organized as follows. In Section 2, we establish the existence and uniqueness of a periodic solution for the system (1.1) by using coincidence degree theory. In Section 3, we establish the global asymptotic stability of a periodic solution for the system (1.1) by constructing a proper Lyapunov functional. Finally, a example is given to demonstrate the effectiveness of our results on global asymptotic stability.

\section{Existence and uniqueness of a periodic solution}

In this section, we first establish the existence of a periodic solution by applying coincidence degree theory. To establish the existence of at least a periodic solution by applying coincidence degree theory, we recall some basic tools in the frame work of Mawhin's coincidence degree [14] that will be used to investigate the existence of periodic solutions.

Let $X, Z$ be Banach spaces, $L$ : Dom $L \subset X \rightarrow Z$ be a linear mapping and $N: X \rightarrow Z$ be a continuous mapping. The mapping $L$ will be called a Fredholm mapping of index zero if $\operatorname{dim} \operatorname{Ker} L=\operatorname{codim} \operatorname{Im} L<\infty$ and $\operatorname{Im} L$ is closed in $Z$. If $L$ is a Fredholm mapping of index zero, then there exist continuous projectors $P: X \rightarrow X$ and $Q: Z \rightarrow Z$ such that $\operatorname{Im} P=\operatorname{Ker} L$ and $\operatorname{Im} L=\operatorname{Ker} Q=\operatorname{Im}(I-Q)$. It follows that $L / \operatorname{Dom} L \cap \operatorname{Ker} P:(I-P) X \rightarrow \operatorname{Im} L$ is invertible. We denote the inverse of the map $L / \operatorname{Dom} L \cap \operatorname{Ker} P$ by $K_{p}$. If $\Omega$ is an open bounded subset of $X$, the mapping $N$ will be called $L$-compact on $\bar{\Omega}$ if $(Q N)(\bar{\Omega})$ is bounded and $K_{p}(I-Q) N: \bar{\Omega} \rightarrow X$ is compact. Since $\operatorname{Im} Q$ is isomorphic to $\operatorname{Ker} L$, there exists an isomorphism $J: \operatorname{Im} Q \rightarrow \operatorname{Ker} L$.

In the proof of our existence theorem, we will use the continuation theorem of Gaines and Mawhin ([14]).

Lemma 2.1 (Continuation Theorem). Let $L$ be a Fredholm mapping of index zero and let $N$ be L-compact on $\bar{\Omega}$. Suppose

(a) $L x \neq \lambda N(x), \forall \lambda \in(0,1), x \in \partial \Omega$;

(b) $Q N(x) \neq 0, \forall x \in \operatorname{Ker} L \cap \partial \Omega$;

(c) $\operatorname{deg}(J Q N x, \Omega \cap \operatorname{Ker} L, 0) \neq 0$.

Then $L x=N x$ has at least one solution in $\operatorname{Dom} L \cap \bar{\Omega}$.

For the sake of convenience, we introduce some notations as follows: $|\cdot|$ denotes the norm in $\mathbb{R}, \bar{f}=\max _{0 \leq t \leq \omega}|f(t)|, \underline{f}=\min _{0 \leq t \leq \omega}|f(t)|$, where $f(t)$ is a continuously periodic function with common period $\omega$.

Our main result on the existence and uniqueness of a periodic solution for the system (1.1) is stated in the following theorem.

Theorem 2.1. We assume that the following conditions hold:

(i) $a_{i}(t), b_{j}(t), h_{i j}(t), w_{j i}(t), \tau_{i j}(t), \sigma_{j i}(t), I_{i}(t), J_{j}(t)$ are continuously periodic functions on $t \in[0,+\infty)$ with common period $\omega>0, i=1,2, \ldots, n ; j=$ $1,2, \ldots, m$; 

$\mathbb{R}$,

(ii) There exist positive constants $A_{j}(j=1,2, \ldots, m)$ such that for $\forall x, y \in$

$$
\left|f_{j}(x)-f_{j}(y)\right| \leq A_{j}|x-y|
$$

(iii) There exist positive constants $B_{i}(i=1,2, \ldots, n)$ such that for $\forall x, y \in \mathbb{R}$,

$$
\left|g_{i}(x)-g_{i}(y)\right| \leq B_{i}|x-y| ;
$$

(iv) There exist two positive constants $l_{i}>1(i=1,2)$ with

$$
a_{i}>\sum_{j=1}^{m} \overline{h_{i j}} A_{j} \lambda_{j} l_{1} \text { and } b_{j}>\sum_{i=1}^{n} \overline{w_{j i}} B_{i} \mu_{i} l_{2}
$$

such that $0<\frac{1}{1-\tau_{i j}^{\prime}}<l_{1}$ and $0<\frac{1}{1-\sigma_{j i}^{\prime}}<l_{2}, i=1,2, \ldots, n, j=1,2, \ldots, m$.

Then the system (1.1) has one unique $\omega$-periodic solution.

Proof. Part 1. The proof of existence of a periodic solution. In order to apply Lemma 2.1 to the system (1.1). Let

$$
X=\left\{u=\left(u_{1}, u_{2}, \ldots, u_{n}, v_{1}, v_{2}, \ldots, v_{m}\right)^{T} \in C\left(\mathbb{R}, \mathbb{R}^{m+n}\right): u(t+\omega)=u(t)\right\}
$$

and

$$
Z=\left\{z \in C\left(\mathbb{R}, \mathbb{R}^{m+n}\right): z(t+\omega)=z(t)\right\}
$$

Define

$$
\|u\|=\max _{t \in[0, \omega]} \sum_{i=1}^{n}\left|u_{i}(t)\right|+\max _{t \in[0, \omega]} \sum_{j=1}^{m}\left|v_{j}(t)\right|, u \in X \quad \text { or } Z .
$$

Equipped with the above norm $\|\cdot\|, X$ and $Z$ are Banach spaces.

Let for $u \in X$,

$$
\begin{aligned}
& N u=\left(\begin{array}{c}
H_{i}(t) \\
K_{j}(t)
\end{array}\right)=\left(\begin{array}{c}
-a_{i}(t) u_{i}(t)+\sum_{j=1}^{m} h_{i j}(t) f_{j}\left(\lambda_{j} v_{j}\left(t-\tau_{i j}(t)\right)+I_{i}(t)\right] \\
-b_{j}(t) v_{j}(t)+\sum_{i=1}^{n} w_{j i}(t) g_{i}\left(\mu_{i} u_{i}\left(t-\sigma_{j i}(t)\right)+J_{j}(t)\right]
\end{array}\right), \\
& L u=u^{\prime}=\frac{\mathrm{d} u(t)}{\mathrm{d} t}, \quad P u=\frac{1}{\omega} \int_{0}^{\omega} u(t) \mathrm{d} t, \quad u \in X, \quad Q z=\frac{1}{\omega} \int_{0}^{\omega} z(t) \mathrm{d} t, \quad z \in Z .
\end{aligned}
$$

Then it follows that $\operatorname{Ker} L=\mathbb{R}^{m+n}, \operatorname{Im} L=\left\{z \in Z: \int_{0}^{\omega} z(t) d t=0\right\}$ is closed in $Z, \operatorname{dim} \operatorname{Ker} L=m+n=\operatorname{codimIm} L$ and $P, Q$ are continuous projectors such that

$$
\operatorname{Im} P=\operatorname{Ker} L, \quad \operatorname{Ker} Q=\operatorname{Im} L=\operatorname{Im}(I-Q) .
$$

Hence, $L$ is a Fredholm mapping of index zero. Furthermore, the generalized inverse (to $L$ ) $K_{p}: \operatorname{Im} L \rightarrow \operatorname{Ker} P \cap \operatorname{Dom} L$ is given by

$$
K_{p}(z)=\int_{0}^{t} z(s) \mathrm{d} s-\frac{1}{\omega} \int_{0}^{\omega} \int_{0}^{s} z(t) \mathrm{d} t \mathrm{~d} s .
$$


Then

and

$$
Q N u=\left(\begin{array}{c}
\frac{1}{\omega} \int_{0}^{\omega} H_{1}(s) \mathrm{d} s \\
\frac{1}{\omega} \int_{0}^{\omega} H_{2}(s) \mathrm{d} s \\
\vdots \\
\frac{1}{\omega} \int_{0}^{\omega} H_{n}(s) \mathrm{d} s \\
\frac{1}{\omega} \int_{0}^{\omega} K_{1}(s) \mathrm{d} s \\
\frac{1}{\omega} \int_{0}^{\omega} K_{2}(s) \mathrm{d} s \\
\vdots \\
\frac{1}{\omega} \int_{0}^{\omega} K_{m}(s) \mathrm{d} s
\end{array}\right)
$$

$$
K_{p}(I-Q) N u=\left(\begin{array}{c}
f_{0}^{t} H_{1}(s) \mathrm{d} s-\frac{1}{\omega} \int_{0}^{\omega} \int_{0}^{t} H_{1}(s) \mathrm{d} s \mathrm{~d} t+\left(\frac{1}{2}-\frac{t}{\omega}\right) \int_{0}^{\omega} H_{1}(s) \mathrm{d} s \\
f_{0}^{t} H_{2}(s) \mathrm{d} s-\frac{1}{\omega} \int_{0}^{\omega} \int_{0}^{t} H_{2}(s) \mathrm{d} s \mathrm{~d} t+\left(\frac{1}{2}-\frac{t}{\omega}\right) \int_{0}^{\omega} H_{2}(s) \mathrm{d} s \\
\vdots \\
f_{0}^{t} H_{n}(s) \mathrm{d} s-\frac{1}{\omega} \int_{0}^{\omega} \int_{0}^{t} H_{n}(s) \mathrm{d} s \mathrm{~d} t+\left(\frac{1}{2}-\frac{t}{\omega}\right) \int_{0}^{\omega} H_{n}(s) \mathrm{d} s \\
f_{0}^{t} K_{1}(s) \mathrm{d} s-\frac{1}{\omega} \int_{0}^{\omega} \int_{0}^{t} K_{1}(s) \mathrm{d} s \mathrm{~d} t+\left(\frac{1}{2}-\frac{t}{\omega}\right) \int_{0}^{\omega} K_{1}(s) \mathrm{d} s \\
\vdots \\
f_{0}^{t} K_{m}(s) \mathrm{d} s-\frac{1}{\omega} \int_{0}^{\omega} \int_{0}^{t} K_{m}(s) \mathrm{d} s \mathrm{~d} t+\left(\frac{1}{2}-\frac{t}{\omega}\right) \int_{0}^{\omega} K_{m}(s) \mathrm{d} s
\end{array}\right) .
$$

Obviously, $Q N$ and $K_{P}(I-Q) N$ are continuous. It is not difficult to show that $K_{p}(I-Q) N(\bar{\Omega})$ is compact for any open bounded set $\Omega \subset X$ by using the Arzela-Ascoli theorem. Moreover, $Q N(\bar{\Omega})$ is clearly bounded. Thus, $N$ is $L$-compact on $\bar{\Omega}$ with any open bounded set $\Omega \subset X$.

The conditions (i) and (ii) in Theorem 2.1 imply that $\forall x \in \mathbb{R}, i=1,2, \ldots, n$, $j=1,2, \ldots, m$,

$$
\left|f_{j}(x)\right| \leq A_{j}|x|+\left|f_{j}(0)\right|
$$

and

$$
\left|g_{i}(x)\right| \leq B_{i}|x|+\left|g_{i}(0)\right| .
$$

Corresponding the operator equation $L x=\lambda N x, \lambda \in(0,1)$, we have for $i=$ $1,2, \ldots, n ; j=1, \ldots, m$,

$$
\left\{\begin{array}{l}
\frac{\mathrm{d} u_{i}(t)}{\mathrm{d} t}=\lambda H_{i}(t) \\
\frac{\mathrm{d} v_{j}(t)}{\mathrm{d} t}=\lambda K_{j}(t) .
\end{array}\right.
$$

Assume that $u \in X$ is a solution of the system (2.3) for some $\lambda \in(0,1)$. Multiplying the first equation of the system $(2.3)$ by $u_{i}(t)$ and integrating over $[0, \omega]$, we have

$$
\left.\left.\int_{0}^{\omega} u_{i}(t)\left\{-a_{i}(t) u_{i}(t)\right)+\sum_{j=1}^{m} h_{i j}(t) f_{j}\left(\lambda_{j} v_{j}\left(t-\tau_{i j}(t)\right)\right)+I_{i}(t)\right]\right\} d t=0 .
$$


Multiplying the second equation of the system (2.3) by $v_{j}(t)$ and integrating over $[0, \omega]$, we have

$$
\left.\int_{0}^{\omega} v_{j}(t)\left\{-b_{j}(t) v_{j}(t)+\sum_{i=1}^{n} w_{j i}(t) g_{i}\left(\mu_{i} u_{i}\left(t-\sigma_{j i}(t)\right)\right)+J_{j}(t)\right]\right\} d t=0 .
$$

By conditions (2.1) and (2.2), from (2.4) and (2.5), we obtain

$$
\underline{a_{i}} \int_{0}^{\omega}\left|u_{i}(t)\right|^{2} d t \leq \int_{0}^{\omega}\left|u_{i}(t)\right|\left\{\sum_{j=1}^{m} \overline{h_{i j}}\left(A_{j} \lambda_{j}\left|v_{j}\left(t-\tau_{i j}(t)\right)\right|+\left|f_{j}(0)\right|\right)+\overline{I_{i}}\right\} d t
$$

and

$$
\underline{b_{j}} \int_{0}^{\omega}\left|v_{j}(t)\right|^{2} d t \leq \int_{0}^{\omega}\left|v_{j}(t)\right|\left\{\sum_{i=1}^{n} \overline{w_{j i}}\left(B_{i} \mu_{i}\left|u_{i}\left(t-\sigma_{j i}(t)\right)\right|+\left|g_{i}(0)\right|\right)+\overline{J_{j}}\right\} d t .
$$

Hence

$$
\begin{aligned}
\underline{a_{i}} \int_{0}^{\omega}\left|u_{i}(t)\right|^{2} d t \leq & \left(\int_{0}^{\omega}\left|u_{i}(t)\right|^{2} d t\right)^{\frac{1}{2}}\left\{\sum _ { j = 1 } ^ { m } \overline { h _ { i j } } \left[A_{j} \lambda_{j}\left(\int_{0}^{\omega}\left|v_{j}\left(t-\tau_{i j}(t)\right)\right|^{2} d t\right)^{\frac{1}{2}}\right.\right. \\
& \left.\left.+\sqrt{\omega}\left|f_{j}(0)\right|\right]+\sqrt{\omega} \overline{I_{i}}\right\}
\end{aligned}
$$

and

$$
\begin{aligned}
\underline{b_{j}} \int_{0}^{\omega}\left|v_{j}(t)\right|^{2} d t \leq & \left(\int_{0}^{\omega}\left|v_{j}(t)\right|^{2} d t\right)^{\frac{1}{2}}\left\{\sum _ { i = 1 } ^ { n } \overline { w _ { j i } } \left[B_{i} \mu_{i}\left(\int_{0}^{\omega}\left|u_{i}\left(t-\sigma_{j i}(t)\right)\right|^{2} d t\right)^{\frac{1}{2}}\right.\right. \\
& \left.\left.+\sqrt{\omega}\left|g_{i}(0)\right|\right]+\sqrt{\omega} \overline{J_{j}}\right\} .
\end{aligned}
$$

That is

$$
\begin{aligned}
\underline{a_{i}}\left(\int_{0}^{\omega}\left|u_{i}(t)\right|^{2} d t\right)^{\frac{1}{2}} \leq & \sum_{j=1}^{m} \overline{h_{i j}}\left[A_{j} \lambda_{j}\left(\int_{0}^{\omega}\left|v_{j}\left(t-\tau_{i j}(t)\right)\right|^{2} d t\right)^{\frac{1}{2}}\right. \\
& \left.+\sqrt{\omega}\left|f_{j}(0)\right|\right]+\sqrt{\omega} \overline{I_{i}}
\end{aligned}
$$

and

$$
\begin{aligned}
\underline{b_{j}}\left(\int_{0}^{\omega}\left|v_{j}(t)\right|^{2} d t\right)^{\frac{1}{2}} \leq & \sum_{i=1}^{n} \overline{w_{j i}}\left[B_{i} \mu_{i}\left(\int_{0}^{\omega}\left|u_{i}\left(t-\sigma_{j i}(t)\right)\right|^{2} d t\right)^{\frac{1}{2}}\right. \\
& \left.+\sqrt{\omega}\left|g_{i}(0)\right|\right]+\sqrt{\omega} \overline{J_{j}} .
\end{aligned}
$$

Denoting $s=t-\tau_{i j}(t)=g(t), \sigma=t-\sigma_{j i}(t)=h(t)$, then

$$
\int_{0}^{\omega} \mid v_{j}\left(t-\tau_{i j}(t) \mid d t=\int_{0}^{\omega} \frac{\left|v_{j}(s)\right|}{1-\tau_{i j}^{\prime}\left(g^{-1}(s)\right)} d s\right.
$$




$$
\begin{gathered}
\int_{0}^{\omega}\left|u_{i}\left(t-\sigma_{j i}(t)\right)\right| d t=\int_{0}^{\omega} \frac{\left|u_{i}(\sigma)\right|}{1-\sigma_{j i}^{\prime}\left(h^{-1}(\sigma)\right)} d \sigma \\
\left(\int_{0}^{\omega}\left|v_{j}\left(t-\tau_{i j}(t)\right)\right|^{2} d t\right)^{\frac{1}{2}}=\left(\int_{0}^{\omega} \frac{\left|v_{j}(s)\right|^{2}}{1-\tau_{i j}^{\prime}\left(g^{-1}(s)\right)} d s\right)^{\frac{1}{2}}
\end{gathered}
$$

and

$$
\left(\int_{0}^{\omega}\left|u_{i}\left(t-\sigma_{j i}(t)\right)\right|^{2} d t\right)^{\frac{1}{2}}=\left(\int_{0}^{\omega} \frac{\left|u_{i}(\sigma)\right|^{2}}{1-\sigma_{j i}^{\prime}\left(h^{-1}(\sigma)\right)} d \sigma\right)^{\frac{1}{2}} .
$$

Applying condition (iv), substituting (2.13) into (2.9) and substituting (2.12) into (2.8) give for $i=1, \ldots, n ; j=1, \ldots, m$,

$$
\begin{aligned}
\underline{a_{i}\left(\int_{0}^{\omega}\left|u_{i}(t)\right|^{2} d t\right)^{\frac{1}{2}} \leq} & \sum_{j=1}^{m} \overline{h_{i j}} A_{j} \lambda_{j} \sqrt{l_{1}}\left(\int_{0}^{\omega}\left|v_{j}(t)\right|^{2} d t\right)^{\frac{1}{2}} \\
& +\sqrt{\omega}\left(\sum_{j=1}^{m} \overline{h_{i j}}\left|f_{j}(0)\right|+\overline{I_{i}}\right)
\end{aligned}
$$

and

$$
\begin{aligned}
\underline{b_{j}}\left(\int_{0}^{\omega}\left|y_{j}(t)\right|^{2} d t\right)^{\frac{1}{2}} \leq & \sum_{i=1}^{n} \overline{w_{j i}} B_{i} \mu_{i} \sqrt{l_{2}}\left(\int_{0}^{\omega}\left|u_{i}(t)\right|^{2} d t\right)^{\frac{1}{2}} \\
& +\sqrt{\omega}\left(\sum_{i=1}^{n} \overline{w_{j i}}\left|g_{i}(0)\right|+\overline{J_{j}}\right) .
\end{aligned}
$$

Denoting for the sake of convenience

$$
\max _{1 \leq i \leq n}\left\{\left(\int_{0}^{\omega}\left|u_{i}(t)\right|^{2} d t\right)^{\frac{1}{2}}\right\}=\left(\int_{0}^{\omega}\left|u_{i}(t)\right|^{2} d t\right)^{\frac{1}{2}}
$$

and

$$
\max _{1 \leq j \leq m}\left\{\left(\int_{0}^{\omega}\left|v_{j}(t)\right|^{2} d t\right)^{\frac{1}{2}}\right\}=\left(\int_{0}^{\omega}\left|v_{j}(t)\right|^{2} d t\right)^{\frac{1}{2}} .
$$

For (2.14) and (2.15), we consider two possible cases:

$$
\begin{aligned}
& \text { (i) }\left(\int_{0}^{\omega}\left|v_{j}(t)\right|^{2} d t\right)^{\frac{1}{2}} \leq\left(\int_{0}^{\omega}\left|u_{i}(t)\right|^{2} d t\right)^{\frac{1}{2}} \text {; } \\
& \text { (ii) }\left(\int_{0}^{\omega}\left|v_{j}(t)\right|^{2} d t\right)^{\frac{1}{2}}>\left(\int_{0}^{\omega}\left|u_{i}(t)\right|^{2} d t\right)^{\frac{1}{2}} \text {. }
\end{aligned}
$$

(i) When $\left(\int_{0}^{\omega}\left|v_{j}(t)\right|^{2} d t\right)^{\frac{1}{2}} \leq\left(\int_{0}^{\omega}\left|u_{i}(t)\right|^{2} d t\right)^{\frac{1}{2}}$, from (2.14), we have

$$
\left(\underline{a_{i}}-\sum_{j=1}^{m} \overline{h_{i j}} A_{j} \lambda_{j} \sqrt{l_{1}}\right)\left(\int_{0}^{\omega}\left|u_{i}(t)\right|^{2} d t\right)^{\frac{1}{2}} \leq \sqrt{\omega}\left(\sum_{j=1}^{m} \overline{h_{i_{0} j}}\left|f_{j}(0)\right|+\overline{I_{i_{0}}}\right),
$$



from which and condition (iv), it follows that

$$
\left(\int_{0}^{\omega}\left|u_{i}(t)\right|^{2} d t\right)^{\frac{1}{2}} \leq \max _{1 \leq i \leq n}\left\{\frac{\sqrt{\omega}\left(\sum_{j=1}^{m} \overline{h_{i j}}\left|f_{j}(0)\right|+\overline{I_{i}}\right)}{\underline{a_{i}}-\sum_{j=1}^{m} \overline{h_{i j}} A_{j} \lambda_{j} \sqrt{l_{1}}}\right\} \stackrel{\text { def }}{=} d_{1} .
$$

Therefore

$$
\left(\int_{0}^{\omega}\left|v_{j}(t)\right|^{2} d t\right)^{\frac{1}{2}} \leq\left(\int_{0}^{\omega}\left|u_{i}(t)\right|^{2} d t\right)^{\frac{1}{2}} \leq d_{1} .
$$

(ii) When $\left(\int_{0}^{\omega}\left|v_{j}(t)\right|^{2} d t\right)^{\frac{1}{2}}>\left(\int_{0}^{\omega}\left|u_{i}(t)\right|^{2} d t\right)^{\frac{1}{2}}$, from (2.15), we have

$$
\left(\int_{0}^{\omega}\left|v_{j}(t)\right|^{2} d t\right)^{\frac{1}{2}} \leq \max _{1 \leq j \leq m}\left\{\frac{\sqrt{\omega}\left(\sum_{i=1}^{n} \overline{w_{j i}}\left|g_{i}(0)\right|+\overline{J_{j}}\right)}{\underline{b_{j}}-\sum_{i=1}^{n} \overline{w_{j i}} B_{i} \mu_{i} \sqrt{l_{2}}}\right\} \stackrel{\text { def }}{=} d_{2} .
$$

Therefore

$$
\left(\int_{0}^{\omega}\left|u_{i}(t)\right|^{2} d t\right)^{\frac{1}{2}} \leq\left(\int_{0}^{\omega}\left|v_{j}(t)\right|^{2} d t\right)^{\frac{1}{2}} \leq d_{2} .
$$

Then from (2.16) and (2.17), we obtain for $i=1,2, \ldots, n, j=1,2, \ldots, m$,

$$
\left(\int_{0}^{\omega}\left|u_{i}(t)\right|^{2} d t\right)^{\frac{1}{2}}<\max \left\{d_{1}, d_{2}\right\} \stackrel{\text { def }}{=} d
$$

and

$$
\left(\int_{0}^{\omega}\left|v_{j}(t)\right|^{2} d t\right)^{\frac{1}{2}}<\max \left\{d_{1}, d_{2}\right\}=d .
$$

From the first equation of the system (2.3), (2.10), (2.18) and (2.19), it follows that

$$
\begin{aligned}
\text { (2.20) } & \int_{0}^{\omega}\left|u_{i}^{\prime}(t)\right| d t \\
\leq & \overline{a_{i}} \int_{0}^{\omega}\left|u_{i}(t)\right| d t+\sum_{j=1}^{m} \overline{h_{i j}} A_{j} \lambda_{j}\left(\int_{0}^{\omega}\left|v_{j}\left(t-\tau_{i j}(t)\right)\right| d t+\omega\left|f_{j}(0)\right|\right)+\omega \overline{I_{i}} \\
\leq & \overline{a_{i}} \sqrt{\omega}\left(\int_{0}^{\omega}\left|u_{i}(t)\right|^{2} d t\right)^{\frac{1}{2}}+\sum_{j=1}^{m} \overline{h_{i j}} A_{j} \lambda_{j} l_{1} \sqrt{\omega}\left(\int_{0}^{\omega}\left|v_{j}(t)\right|^{2} d t\right)^{\frac{1}{2}} \\
& +\omega\left(\sum_{j=1}^{m} \overline{h_{i j}}\left|f_{j}(0)\right|+\overline{I_{i}}\right) \\
< & \overline{a_{i}} \sqrt{\omega} d+\sqrt{\omega} \sum_{j=1}^{m} \overline{h_{i j}} A_{j} \lambda_{j} l_{1} \sqrt{\omega} d+\omega\left(\sum_{j=1}^{m} \overline{h_{i j}}\left|f_{j}(0)\right|+\overline{I_{i}}\right) \stackrel{\text { def }}{=} c_{1} .
\end{aligned}
$$


Similarly, from the second equation of the system (2.3), (2.11), (2.18) and (2.19), it follows that there exists a positive constant $c_{2}$ such that

$$
\int_{0}^{\omega}\left|v_{j}^{\prime}(t)\right| d t<c_{2}
$$

From (2.18) and (2.19), it follows that there exist points $t_{i}$ and $\overline{t_{j}}$ such that

$$
\left|u_{i}\left(t_{i}\right)\right|<\frac{d}{\sqrt{\omega}}
$$

and

$$
\left|v_{j}\left(\overline{t_{j}}\right)\right| \leq \frac{d}{\sqrt{\omega}}
$$

Since for $\forall t \in[0, \omega]$,

$$
\left|u_{i}(t)\right| \leq\left|u_{i}\left(t_{i}\right)\right|+\int_{0}^{\omega}\left|u_{i}^{\prime}(t)\right| d t
$$

and

$$
\left|v_{j}(t)\right| \leq\left|v_{j}\left(\overline{t_{j}}\right)\right|+\int_{0}^{\omega}\left|v_{j}^{\prime}(t)\right| d t
$$

then from (2.20)-(2.23), we have

$$
\left|u_{i}(t)\right| \leq \frac{d}{\sqrt{\omega}}+c_{1}
$$

and

$$
\left|v_{j}(t)\right| \leq \frac{d}{\sqrt{\omega}}+c_{2}
$$

Obviously, $\frac{d}{\sqrt{\omega}}, c_{1}, c_{2}$ are all independent of $\lambda$. Now let

$$
\begin{aligned}
\Omega=\left\{u=\left(u_{1}, u_{2}, \ldots, u_{n} ; v_{1}, v_{2}, \ldots, v_{m}\right)^{T} \in X:\right. \\
\left.\|u\|<n\left(\frac{d}{\sqrt{\omega}}+r_{1}+c_{1}\right)+m\left(\frac{d}{\sqrt{\omega}}+r_{2}+c_{2}\right)\right\},
\end{aligned}
$$

where $r_{1}, r_{2}$ are two chosen positive constants such that the bound of $\Omega$ is larger. Then $\Omega$ are bounded open subsets of $X$. Hence $\Omega$ satisfies the requirement (a) in Lemma 2.1. Next we prove that (b) in Lemma 2.1 holds. If it is not true, then when $u \in \partial \Omega \cap \operatorname{Ker} L=\partial \Omega \cap \mathbb{R}^{m+n}$, we have

$$
\begin{aligned}
& Q N u \\
= & \left(\frac{1}{\omega} \int_{0}^{\omega} H_{1}(t) d t, \frac{1}{\omega} \int_{0}^{\omega} H_{2}(t) d t, \ldots, \frac{1}{\omega} \int_{0}^{\omega} H_{n}(t) d t ; \frac{1}{\omega} \int_{0}^{\omega} K_{1}(t) d t, \ldots, \frac{1}{\omega} \int_{0}^{\omega} K_{m}(t) d t\right)^{T} \\
= & (0, \ldots, 0)^{T} .
\end{aligned}
$$


Therefore there exist points $\xi_{i}(i=1,2, \ldots, n)$ and $\eta_{j}(j=1,2, \ldots, m)$ such that

$$
\left\{\begin{array}{l}
H_{i}\left(\xi_{i}\right)=0 \\
K_{j}\left(\eta_{j}\right)=0
\end{array}\right.
$$

From this and following the arguments of (2.22) and (2.23), we have for $\forall i=$ $1,2, \ldots, n ; j=1,2, \ldots, m, t \in[0, \omega]$

$$
\left|u_{i}(t)\right|<\frac{d}{\sqrt{\omega}}
$$

and

Hence

$$
\left|v_{j}(t)\right|<\frac{d}{\sqrt{\omega}} .
$$

$$
\|u\|<n \frac{d}{\sqrt{\omega}}+m \frac{d}{\sqrt{\omega}} .
$$

Thus $u \in \Omega \cap \mathbb{R}^{m+n}$. This contradicts the fact that $u \in \partial \Omega \cap \mathbb{R}^{m+n}$. Hence this proves (b) in Lemma 2.1 holds. Finally, we show that (c) in Lemma 2.1 holds. We only need to prove that $\operatorname{deg}\left\{-J Q N u, \Omega \cap \operatorname{Ker} L,(0,0)^{T}\right\} \neq(0,0, \ldots, 0)^{T}$. Now we show that

$$
\begin{aligned}
& \operatorname{deg}\left\{-J Q N u, \Omega \cap \operatorname{Ker} L,(0,0, \ldots, 0)^{T}\right\} \\
= & \operatorname{deg}\left\{\left(\underline{a_{1}} u_{1}, \underline{a_{2}} u_{2}, \ldots, \underline{a_{n}} u_{n} ; \underline{b_{1}} v_{1}, \ldots, \underline{b_{m}} v_{m}\right)^{T}, \Omega \cap \operatorname{Ker} L,(0, \ldots, 0)^{T}\right\} .
\end{aligned}
$$

To this end, we define a mapping $\phi: \operatorname{Dom} L \times[0,1] \rightarrow X$ by

$$
\begin{aligned}
& \phi\left(u_{1}, u_{2}, \ldots, u_{n} ; v_{1}, v_{2}, \ldots, v_{m}, \mu\right) \\
= & -\frac{\mu}{\omega}\left(\int_{0}^{\omega} H_{1}(t) d t, \int_{0}^{\omega} H_{2}(t) d t, \ldots, \int_{0}^{\omega} H_{n}(t) d t, \int_{0}^{\omega} K_{1}(t) d t, \ldots, \int_{0}^{\omega} K_{m}(t) d t\right) \\
& +(1-\mu)\left(\underline{a_{1}} u_{1}, \underline{a_{2}} u_{2}, \ldots, \underline{a_{n}} u_{n} ; \underline{b_{1}} v_{1}, \ldots, \underline{b_{m}} v_{m}\right),
\end{aligned}
$$

where $\mu \in[0,1]$ is a parameter. We show that when $u \in \partial \Omega \cap \operatorname{Ker} L=$ $\partial \Omega \cap \mathbb{R}^{m+n}, \phi\left(u_{1}, u_{2}, \ldots, u_{n} ; v_{1}, \ldots, v_{m}, \mu\right) \neq(0,0, \ldots, 0)^{T}$. If it is not true, then when $u \in \partial \Omega \cap \operatorname{Ker} L=\partial \Omega \cap \mathbb{R}^{m+n}, \phi\left(u_{1}, u_{2}, \ldots, u_{n} ; v_{1}, \ldots, v_{m}, \mu\right)=$ $(0,0, \ldots, 0)^{T}$. Thus constant vector $u$ with $u \in \partial \Omega$ satisfies for $i=1,2, \ldots, n$, $j=1,2, \ldots, m$,

$$
\begin{aligned}
& \frac{\mu}{\omega} \int_{0}^{\omega}\left\{a_{i}(t) u_{i}-\sum_{j=1}^{m} h_{i j}(t) f_{j}\left(\lambda_{j} v_{j}\right)-I_{i}(t)\right\} d t+(1-\mu) \underline{a_{i}} u_{i}=0, \\
& \frac{\mu}{\omega} \int_{0}^{\omega}\left\{b_{j}(t) v_{j}-\sum_{i=1}^{n} w_{j i}(t) g_{i}\left(\mu_{i} u_{i}\right)-J_{j}(t)\right\} d t+(1-\mu) \underline{b_{j}} v_{j}=0 .
\end{aligned}
$$

We make the following claims:

Claim 1. $\left|u_{i}\right|^{*}<\frac{d}{\sqrt{\omega}}+c_{1}+r_{1}$. Otherwise, $\left|u_{i}\right|^{*} \geq \frac{d}{\sqrt{\omega}}+c_{1}+r_{1}$. We consider two possible cases: (a) $\left|v_{j}\right|^{*} \leq\left|u_{i}\right|^{*}$;(b) $\left|v_{j}\right|^{*}>\left|u_{i}\right|^{*}$, where $\left|v_{j}\right|^{*}=$ $\max _{1 \leq j \leq m}\left\{\left|v_{j}\right|\right\},\left|u_{i}\right|^{*}=\max _{1 \leq i \leq n}\left\{\left|u_{i}\right|\right\}$. 
(a) When $\left|v_{j}\right|^{*} \leq\left|u_{i}\right|^{*}$, we have

$$
\begin{aligned}
& \frac{\mu}{\omega} \int_{0}^{\omega} u_{i}\left\{a_{i}(t) u_{i}-\sum_{j=1}^{m} h_{i j}(t) f_{j}\left(\lambda_{j} v_{j}\right)-I_{i}(t)\right\} d t+(1-\mu) \underline{a_{i}} u_{i}^{2} \\
\geq & \mu\left[\underline{a_{i}} u_{i}^{2}-\sum_{j=1}^{m} \overline{h_{i j}}\left|u_{i}\right|\left(\lambda_{j} A_{j}\left|v_{j}\right|+\left|f_{j}(0)\right|\right)-\overline{I_{i}}\left|u_{i}\right|\right]+(1-\mu) \underline{a_{i}} u_{i}^{2} \\
\geq & \underline{a_{i}}\left|u_{i}\right|^{2}-\sum_{j=1}^{m} \overline{h_{i j}}\left(\lambda_{j} A_{j}\left|v_{j}\right|\left|u_{i}\right|+\left|u_{i}\right|\left|f_{j}(0)\right|\right)-\overline{I_{i}}\left|u_{i}\right| .
\end{aligned}
$$

Then

$$
\begin{aligned}
& \underline{a_{i}}\left(\left|u_{i}\right|^{*}\right)^{2}-\sum_{j=1}^{m} \overline{h_{i j}}\left(A_{j} \lambda_{j}\left|u_{i}\right|\left|v_{j}\right|+\left|u_{i}\right|\left|f_{j}(0)\right|\right)-\overline{I_{i}}\left|u_{i}\right|^{*} \\
> & \underline{a_{i}}\left(\left|u_{i}\right|^{*}\right)^{2}-\sum_{j=1}^{m} \overline{h_{i j}}\left(A_{j} \lambda_{j}\left|u_{i}\right|^{*}\left|u_{i}\right|^{*}+\left|u_{i}\right|^{*}\left|f_{j}(0)\right|\right)-\overline{I_{i}}\left|u_{i}\right|^{*} \\
> & \left|u_{i}\right|^{*}\left\{\left(\underline{a_{i}}-\sum_{j=1}^{m} \overline{h_{i j}} A_{j} \lambda_{j}\right)\left(\frac{d}{\sqrt{\omega}}+c_{1}+r_{1}\right)-\sum_{j=1}^{m} \overline{h_{i j}} A_{j} \lambda_{j}\left|f_{j}(0)\right|-\overline{I_{i}}\right\} \\
> & \left|u_{i}\right|^{*}\left(c_{1}+r_{1}\right)\left(\underline{a_{i}}-\sum_{j=1}^{m} \overline{h_{i j}} A_{j} \lambda_{j}\right) \\
> & 0
\end{aligned}
$$

which contradicts (2.24).

(b) When $\left|v_{j}\right|^{*}>\left|u_{i}\right|^{*}$, we have

$$
\begin{aligned}
& \frac{\mu}{\omega} \int_{0}^{\omega} v_{j}\left\{b_{j}(t) v_{j}-\sum_{i=1}^{n} w_{j i}(t) g_{i}\left(\mu_{i} u_{i}\right)-J_{j}(t)\right\} d t+(1-\mu) \underline{b_{j}} v_{j}^{2} \\
\geq & \mu\left[\underline{b_{j}} v_{j}^{2}-\sum_{i=1}^{n} \overline{w_{j i}}\left|v_{j}\right|\left(\mu_{i} B_{i}\left|u_{i}\right|+\left|g_{i}(0)\right|\right)-\overline{J_{j}}\left|v_{j}\right|\right]+(1-\mu) \underline{b_{j}} v_{j}^{2} \\
\geq & \underline{b_{j}}\left|v_{j}\right|^{2}-\sum_{i=1}^{n} \overline{w_{j i}}\left(\mu_{i} B_{i}\left|v_{j}\right|\left|u_{i}\right|+\left|v_{j}\right|\left|f_{j}(0)\right|\right)-\overline{J_{j}}\left|v_{j}\right| .
\end{aligned}
$$

Then

$$
\begin{aligned}
& \underline{b_{j}}\left(\left|v_{j}\right|^{*}\right)^{2}-\sum_{i=1}^{n} \overline{w_{j i}}\left(B_{i} \mu_{i}\left|u_{i}\right|\left|v_{j}\right|+\left|v_{j}\right|\left|g_{i}(0)\right|\right)-\overline{J_{j}}\left|v_{j}\right|^{*} \\
> & \underline{b_{j}}\left(\left|v_{j}\right|^{*}\right)^{2}-\sum_{i=1}^{n} \overline{w_{j i}}\left(B_{i} \mu_{i}\left|v_{j}\right|^{*}\left|v_{j}\right|^{*}+\left|v_{j}\right|^{*}\left|f_{j}(0)\right|\right)-\overline{J_{j}}\left|v_{j}\right|^{*} \\
> & \left|v_{j}\right|^{*}\left\{\left(\underline{b_{j}}-\sum_{i=1}^{n} \overline{w_{j i}} B_{i} \mu_{i}\right)\left(\frac{d}{\sqrt{\omega}}+c_{2}+r_{2}\right)-\sum_{i=1}^{n} \overline{w_{j i}} B_{i} \mu_{i}\left|g_{i}(0)\right|-\overline{J_{j}}\right\}
\end{aligned}
$$




$$
\begin{aligned}
& >\left|v_{j}\right|^{*}\left(c_{2}+r_{2}\right)\left(\underline{b_{j}}-\sum_{i=1}^{n} \overline{w_{j i}} B_{i} \mu_{i}\right) \\
& >0,
\end{aligned}
$$

which contradicts (2.25). From the arguments of (a) and (b), Claim 1 holds.

Claim 2. $\left|v_{j}\right|^{*} \leq \frac{d}{\sqrt{\omega}}+c_{2}+r_{2}$. Otherwise, $\left|v_{j}\right|^{*}>\frac{d}{\sqrt{\omega}}+c_{2}+r_{2}$. We consider two possible cases: (a) $\left|u_{i}\right|^{*} \leq\left|v_{j}\right|^{*}$; (b) $\left|u_{i}\right|^{*}>\left|v_{j}\right|^{*}$. This arguments of (a) and (b) are similar to this arguments of (b) and (a) in Claim 1, then the proof is omitted. Hence Claim 2 holds. Thus, $\left|u_{i}\right|<\frac{d}{\sqrt{\omega}}+c_{1}+r_{1}, i=1,2, \ldots, n$ and $\left|v_{j}\right|<\frac{d}{\sqrt{\omega}}+c_{2}+r_{2}, j=1,2, \ldots, m$. Then $u \in \Omega \cap \mathbb{R}^{m+n}$. This contradicts the fact $u \in \partial \Omega \cap \mathbb{R}^{m+n}$. According to topological degree theory and by taking $J=I$ since $\operatorname{Ker} L=\operatorname{Im} Q$, we obtain,

$$
\begin{aligned}
& \operatorname{deg}\left\{-J Q N u, \Omega \cap \operatorname{Ker} L,(0,0)^{T}\right\} \\
= & \operatorname{deg}\left\{\phi\left(u_{1}, u_{2}, \ldots, u_{n} ; v_{1}, v_{2}, \ldots, v_{m}, 1\right), \Omega \cap \operatorname{Ker} L,(0,0)^{T}\right\} \\
= & \operatorname{deg}\left\{\phi\left(u_{1}, u_{2}, \ldots, u_{n} ; v_{1}, v_{2}, \ldots, v_{m}, 0\right), \Omega \cap \operatorname{Ker} L,(0,0)^{T}\right\} \\
= & \operatorname{deg}\left\{\left(\underline{a_{1}} u_{1}, \underline{a_{2}} u_{2}, \ldots, \underline{a_{n}} u_{n} ; \underline{b_{1}} v_{1}, \ldots, \underline{b_{m}} v_{m}\right)^{T}, \Omega \cap \operatorname{Ker} L,(0, \ldots, 0)^{T}\right\} \\
\neq & 0 .
\end{aligned}
$$

So far, we have proved that $\Omega$ satisfies all the assumptions in Lemma 2.1. Therefore, the system (1.1) has at least one $\omega$-periodic solutions.

Part 2. The proof of uniqueness of a periodic solution. Let

$$
\begin{aligned}
u & =\left(u_{1}(t), u_{2}(t), \ldots, u_{n}(t) ; v_{1}(t), v_{2}(t), \ldots, v_{m}(t)\right)^{T}, \\
u^{*} & =\left(u_{1}^{*}(t), u_{2}^{*}(t), \ldots, u_{n}^{*}(t) ; v_{1}^{*}(t), v_{2}^{*}(t), \ldots, v_{m}^{*}(t)\right)^{T}
\end{aligned}
$$

be two $\omega$ periodic solutions of the system (1.1). Denote

$$
\begin{aligned}
& \int_{0}^{\omega}\left(u_{i}(t)-u_{i}^{*}(t)\right)^{2} d t=\max _{1 \leq i \leq n}\left\{\int_{0}^{\omega}\left(u_{i}(t)-u_{i}^{*}(t)\right)^{2} d t\right\}, \\
& \int_{0}^{\omega}\left(v_{j}(t)-v_{j}^{*}(t)\right)^{2} d t=\max _{1 \leq j \leq m}\left\{\int_{0}^{\omega}\left(v_{j}(t)-v_{j}^{*}(t)\right)^{2} d t\right\} .
\end{aligned}
$$

From the system (1.1), we have

$$
\begin{aligned}
u_{i}^{\prime}(t)-\left(u_{i}^{*}\right)^{\prime}(t)= & -a_{i}(t)\left(u_{i}(t)-u_{i}^{*}(t)\right) \\
& +\sum_{j=1}^{m} h_{i j}(t)\left\{f_{j}\left(\lambda_{j} v_{j}\left(t-\tau_{i j}(t)\right)\right)-f_{j}\left(\lambda_{j} v_{j}^{*}\left(t-\tau_{i j}(t)\right)\right)\right\}
\end{aligned}
$$

and

$$
\begin{aligned}
v_{j}^{\prime}(t)-\left(v_{j}^{*}\right)^{\prime}(t)= & -b_{j}(t)\left(v_{j}(t)-v_{j}^{*}(t)\right) \\
& +\sum_{i=1}^{n} w_{j i}(t)\left\{g_{i}\left(\mu_{i} u_{i}\left(t-\sigma_{j i}(t)\right)\right)-g_{i}\left(\mu_{i} u_{i}^{*}\left(t-\sigma_{j i}(t)\right)\right)\right\} .
\end{aligned}
$$


Then from conditions (iv), (ii) and (iii), we have

$$
\begin{aligned}
& \underline{a_{i}} \int_{0}^{\omega}\left(u_{i}(t)-u_{i}^{*}(t)\right)^{2} d t \\
& \leq \sum_{j=1}^{m} \overline{h_{i j}} \int_{0}^{\omega} \lambda_{j} A_{j}\left|u_{i}(t)-u_{i}^{*}(t)\right|\left|v_{j}\left(t-\tau_{i j}(t)\right)-v_{j}^{*}\left(t-\tau_{i j}(t)\right)\right| d t \\
& \leq \sum_{j=1}^{m} \overline{h_{i j}} \lambda_{j} A_{j}\left(\int_{0}^{\omega}\left|u_{i}(t)-u_{i}^{*}(t)\right|^{2} d t\right)^{\frac{1}{2}}\left(\int_{0}^{\omega}\left|v_{j}\left(t-\tau_{i j}(t)\right)-v_{j}^{*}\left(t-\tau_{i j}(t)\right)\right|^{2} d t\right)^{\frac{1}{2}} \\
& \leq \sum_{j=1}^{m} \overline{h_{i j}} \lambda_{j} A_{j} \sqrt{l_{1}}\left(\int_{0}^{\omega}\left|u_{i}(t)-u_{i}^{*}(t)\right|^{2} d t\right)^{\frac{1}{2}}\left(\int_{0}^{\omega}\left|v_{j}(t)-v_{j}^{*}(t)\right|^{2} d t\right)^{\frac{1}{2}} \\
& \text { and }(2.27) \\
&\left.\quad \leq b_{j} \int_{0}^{\omega} \overline{\left(v_{j}\right.}(t)-v_{j}^{*}(t)\right)^{2} d t \\
& \quad \leq B_{i} \sqrt{l_{2}}\left(\int_{0}^{\omega}\left|v_{j}(t)-v_{j}^{*}(t)\right|^{2} d t\right)^{\frac{1}{2}}\left(\int_{0}^{\omega}\left|u_{i}(t)-u_{i}^{*}(t)\right|^{2} d t\right)^{\frac{1}{2}} .
\end{aligned}
$$

From (2.26) and (2.27), we have

$$
\begin{aligned}
& \underline{a_{i}}\left(\int_{0}^{\omega}\left(u_{i}(t)-u_{i}^{*}(t)\right)^{2} d t\right)^{\frac{1}{2}} \\
\leq & \sum_{j=1}^{m} \overline{h_{i j}} \lambda_{j} A_{j} \sqrt{l_{1}}\left(\int_{0}^{\omega}\left|v_{j}(t)-v_{j}^{*}(t)\right|^{2} d t\right)^{\frac{1}{2}}
\end{aligned}
$$

and

$$
\begin{aligned}
& \underline{b_{j}}\left(\int_{0}^{\omega}\left(v_{j}(t)-v_{j}^{*}(t)\right)^{2} d t\right)^{\frac{1}{2}} \\
\leq & \sum_{i=1}^{n} \overline{w_{j i}} \mu_{i} B_{i} \sqrt{l_{2}}\left(\int_{0}^{\omega}\left|u_{i}(t)-u_{i}^{*}(t)\right|^{2} d t\right)^{\frac{1}{2}} .
\end{aligned}
$$

Substituting (2.29) into (2.28) gives, for $i=1,2, \ldots, n, j=1,2, \ldots, m$,

$$
\left(\underline{a_{i}}-\sum_{j=1}^{m} \overline{h_{i j}} \lambda_{j} A_{j} \sqrt{l_{1}} \frac{\sum_{i=1}^{n} \overline{w_{j i}} \mu_{i} B_{i} \sqrt{l_{2}}}{\underline{b_{j}}}\right)\left(\int_{0}^{\omega}\left(u_{i}(t)-u_{i}^{*}(t)\right)\right)^{\frac{1}{2}} \leq 0 .
$$

Then for $i=1,2, \ldots, n$,

$$
\left(\int_{0}^{\omega}\left(u_{i}(t)-u_{i}^{*}(t)\right)^{2} d t\right)^{\frac{1}{2}}=0 .
$$

Hence for $i=1,2, \ldots, n$,

$$
u_{i}(t)=u_{i}^{*}(t)
$$


Similarly, substituting (2.28) into (2.29) gives for $j=1,2, \ldots, m$,

$$
v_{j}(t)=v_{j}^{*}(t) .
$$

Therefore the proof of uniqueness is complete. This completes the proof of Theorem 2.1.

\section{Globally asymptotic stability of the unique periodic solution}

In this section, by constructing a Lyapunov functional, we derive new sufficient conditions for the global asymptotic stability of the unique periodic solution of the system (1.1).

Theorem 3.1. In addition to conditions (i), (ii), (iii) in Theorem 2.1, we assume further that the following conditions hold:

$\left(H_{1}\right)$ There exists two positive constants $l_{i} \geq 1(i=1,2)$ with

$$
\begin{aligned}
& \underline{a_{i}}>\max \left\{\sum_{j=1}^{m} \overline{h_{i j}} A j \lambda_{j} l_{1}, \sum_{j=1}^{m} \overline{w_{j i}} \mu_{i} B_{i} l_{2}\right\} \text { and } \\
& \underline{b_{j}}>\max \left\{\sum_{i=1}^{n} \overline{w_{j i}} B_{i} \mu_{i} l_{2}, \sum_{i=1}^{n} \overline{h_{i j}} \lambda_{j} A j l_{1}\right\}
\end{aligned}
$$

such that

$$
\begin{aligned}
& 0<\frac{1}{1-\tau_{i j}^{\prime}}<l_{1}, \\
& 0<\frac{1}{1-\sigma_{j i}^{\prime}}<l_{2} ;
\end{aligned}
$$

$\left(H_{2}\right)$ There exist constants $\sigma_{j i}$ and $\tau_{i j}, i=1,2, \ldots, n ; j=1,2, \ldots, m$, such that

$$
0<\sigma_{i j}(t)<\sigma_{i j}, 0<\tau_{j i}(t)<\tau_{j i} .
$$

Then the unique $\omega$ periodic solution of the system (1.1) is globally asymptotically stable.

Proof. By Theorem 2.1, in view of $\left(H_{1}\right)$, system (1.1) has a unique $\omega$ periodic solution, say, $u^{*}(t)=\left(u_{1}^{*}(t), u_{2}^{*}(t), \ldots, u_{n}^{*}(t) ; v_{1}^{*}(t), v_{2}^{*}(t), \ldots, v_{m}^{*}(t)\right)^{T}$. Suppose that $u(t)=\left(u_{1}(t), u_{2}(t), \ldots, u_{n}(t) ; v_{1}(t), v_{2}(t), \ldots, v_{m}(t)\right)^{T}$ is any $\omega$ periodic solution of the system (1.1) with the initial conditions (1.2).

We define a Lyapunov functional as follows for $t>0, i=1,2, \ldots, n ; j=$ $1,2, \ldots, m$

$$
\begin{aligned}
V\left(u_{i}(t), v_{j}(t)\right)= & \sum_{i=1}^{n}\left|u_{i}(t)-u_{i}^{*}(t)\right|+\sum_{j=1}^{m}\left|v_{j}(t)-v_{j}^{*}(t)\right| \\
& +\sum_{i=1}^{n} \sum_{j=1}^{m} \overline{h_{i j}} \lambda_{j} A_{j} \int_{t-\tau_{i j}(t)}^{t} \frac{\left|v_{j}(\sigma)-v_{j}^{*}(\sigma)\right|}{1-\tau_{i j}^{\prime}\left(g^{-1}(\sigma)\right)} d \sigma
\end{aligned}
$$




$$
+\sum_{i=1}^{n} \sum_{j=1}^{m} \overline{w_{j i}} \mu_{i} B_{i} \int_{t-\sigma_{j i}(t)}^{t} \frac{\left|u_{i}(\sigma)-u_{i}^{*}(\sigma)\right|}{1-\sigma_{j i}^{\prime}\left(h^{-1}(\sigma)\right)} d \sigma
$$

where, $t-\tau_{i j}(t)=g(t), t-\sigma_{j i}(t)=h(t)$.

Calculating the upper right derivative $D^{+} V\left(u_{i}(t), v_{j}(t)\right)$ of $V\left(u_{i}(t), v_{j}(t)\right)$ along the solutions of the system (1.1), we obtain from conditions (ii) and (iii),

$$
\begin{aligned}
& D^{+} V\left(u_{i}(t), v_{j}(t)\right) \\
& =\sum_{i=1}^{n}\left[-a_{i}(t)\left|u_{i}(t)-u_{i}^{*}(t)\right|+\sum_{j=1}^{m} \overline{h_{i j}} \lambda_{j} A_{j}\left|v_{j}\left(t-\tau_{i j}(t)\right)-v_{j}^{*}\left(t-\tau_{i j}(t)\right)\right|\right] \\
& +\sum_{j=1}^{m}\left[-b_{j}(t)\left|v_{j}(t)-v_{j}^{*}(t)\right|+\sum_{i=1}^{n} \overline{w_{j i}} \mu_{i} B_{i}\left|u_{i}\left(t-\sigma_{j i}(t)\right)-u_{i}^{*}\left(t-\sigma_{j i}(t)\right)\right|\right] \\
& +\sum_{i=1}^{n} \sum_{j=1}^{m} \overline{h_{i j}} \lambda_{j} A_{j}\left[\frac{\left|v_{j}(t)-v_{j}^{*}(t)\right|}{1-\tau_{i j}^{\prime}\left(g^{-1}(t)\right)}-\left|v_{j}\left(t-\tau_{i j}(t)\right)-v_{j}^{*}\left(t-\tau_{i j}(t)\right)\right|\right] \\
& +\sum_{i=1}^{n} \sum_{j=1}^{m} \overline{w_{j i}} \mu_{i} B_{i}\left[\frac{\left|u_{i}(t)-u_{i}^{*}(t)\right|}{1-\sigma_{j i}^{\prime}\left(h^{-1}(t)\right)}-\left|u_{i}\left(t-\sigma_{j i}(t)\right)-u_{j}^{*}\left(t-\sigma_{j i}(t)\right)\right|\right] \\
& \leq-\sum_{i=1}^{n}\left(\underline{a_{i}}-\sum_{j=1}^{m} \overline{w_{j i}} \mu_{i} B_{i} l_{2}\right)\left|u_{i}(t)-u_{i}^{*}(t)\right| \\
& \left.-\sum_{j=1}^{m} \underline{\left(b_{j}\right.}-\sum_{i=1}^{n} \overline{h_{i j}} \lambda_{j} A_{j} l_{1}\right)\left|v_{j}(t)-v_{j}^{*}(t)\right| .
\end{aligned}
$$

In view of condition (iv), it follows that $D^{+} V\left(u_{i}(t), v_{j}(t)\right)<0$ for $u_{i}(t) \neq$ $u_{i}^{*}(t), v_{j}(t) \neq v_{j}^{*}(t)$. Hence, by the Lyapunov-type theorem in functional differential equations we can conclude that the unique $\omega$ periodic solution of the system (1.1) is globally asymptotically stable.

\section{An example}

Example 1. Consider the following BAM neural networks with time-varying delays:

$$
\begin{aligned}
& \frac{\mathrm{d} u_{1}(t)}{\mathrm{d} t}=-(9+\sin t) u_{1}(t)+v_{1}\left[t-\left(1+\frac{\sin t}{2}\right)\right]+\sin t, \\
& \frac{\mathrm{d} v_{1}(t)}{\mathrm{d} t}=-(8+\cos t) v_{1}(t)+u_{1}\left[t-\left(1+\frac{\sin t}{3}\right)\right]+\cos t .
\end{aligned}
$$

In Theorem 3.1, $A_{1}=1, B_{1}=1, l_{1}=2, l_{2}=3, \tau_{11}=\frac{3}{2}, \sigma_{11}=\frac{4}{3}, \overline{h_{11}}=1$, $\overline{w_{11}}=1, \lambda_{1}=\mu_{1}=1, \underline{a_{1}}=\underline{b_{1}}=2$. 
It is easy to prove that all conditions in Theorem 3.1 are satisfied. By Theorem 3.1, the system (4.1) has a unique $\omega$ periodic solution which is globally asymptotically stable.

\section{References}

[1] S. Arik, Global asymptotic stability analysis of bidirectional associative memory neural networks with time delays, IEEE Trans. Neural Networks 16 (2005), no. 3, 580-586.

[2] S. Arik and V. Tavsanoglu, Global asymptotic stability analysis of bidirectional associative memory neural networks with constant time delays, Neurocomputing 68 (2005), 161-176.

[3] J. D. Cao, Global asymptotic stability of delayed bi-directional associative memory neural networks, Appl. Math. Comput. 142 (2003), no. 2-3, 333-339.

[4] J. D. Cao and Q. H. Jiang, An analysis of periodic solutions of bi-directional associative memory networks with time-varying delays, Phys. Lett. A 330 (2004), no. 3-4, 203-213.

[5] J. D. Cao and J. Q. Lu, Adaptive synchronization of neural networks with or without time-varying delay, Chaos 16 (2006), no. 1, 013133, 6 pp.

[6] J. D. Cao and J. Wang, Global exponential stability and periodicity of recurrent neural networks with time delays, IEEE Trans. Circuits Syst. I Regul. Pap. 52 (2005), no. 5, 920-931.

[7] J. D. Cao and L. Wang, Periodic oscillatory solution of bidirectional associative memory networks with delays, Phys. Rev. E (3) 61 (2000), no. 2, 1825-1828.

[8] _ Exiponential stability and periodic oscillatory solution in BAM networks with delays, IEEE Trans. Neural Networks 13 (2002), no. 2, 457-463.

[9] J. D. Cao, K. Yuan, D. W. C. Ho, and J. Lam, Global point dissipativity of neural networks with mixed time-varying delays, Chaos 16 (2006), no. 1, 013105, 9 pp.

[10] A. P. Chen and F. L. Chen, Periodic solution to BAM neural network with delays on time scales, Neurocomputing 73 (2009), no. 1-3, 274-282.

[11] A. P. Chen, L. H. Huang, and J. D. Cao, Existence and stability of almost periodic solution for BAM neural networks with delays, Appl. Math. Comput. 137 (2003), no. 1, 177-193.

[12] A. P. Chen, L. H. Huang, Z. G. Liu, and J. D. Cao, Periodic bidirectional associative memory neural networks with distributed delays, J. Math. Anal. Appl. 317 (2006), no. $1,80-102$.

[13] C. H. Feng and R. Plamondon, Stability analysis of bidirectional associative memory networks with time delays, IEEE Trans. Neural Networks 14 (2003), no. 6, 1560-1565.

[14] R. E. Gaines and J. L. Mawhin, Coincidence Degree, and Nonlinear Differential Equations, Lecture Notes in Mathematics, Vol. 568. Springer-Verlag, Berlin-New York, 1977.

[15] K. Gopalsamy and X. Z. He, Delay-independent stability in bidirectional associative memory networks, IEEE Trans. Neural Networks 5 (1994), no. 6, 998-1002.

[16] B. Kosko, Bidirectional associative memories, IEEE Trans. Systems Man Cybernet. 18 (1988), no. 1, 49-60

[17] C. D. Li, X. F. Liao, and R. Zhang, Delay-dependent exponential stability analysis of bi-directional associative memory neural networks with time delay: an LMI approach, Chaos, Solitons Fractals 24 (2005), no. 4, 1119-1134.

[18] J. L. Liang and J. D. Cao, Exponential stability of continuous-time and discrete-time bidirectional associative memory networks with delays, Chaos, Solitons Fractals 22 (2004), no. 4, 773-785.

[19] X. F. Liao, K. W. Wong, and S. Z. Yuan, Convergence dynamics of hybrid bidirectional associative memory neural networks with distributed delays, Phys. Lett. A 316 (2003), no. $1-2,55-64$. 
[20] Z. G. Liu, A. P. Chen, J. D. Cao, and L. H. Huang, Existence and global exponential stability of periodic solution for BAM neural networks with periodic coefficients and time-varying delays, IEEE Trans. Circuits Systems I Fund. Theory Appl. 50 (2003), no. $9,1162-1173$

[21] Z. G. Liu, A. P. Chen, and L. H. Huang, Existence and global exponential stability of periodic solution to self-connection BAM neural networks with delays, Phys. Lett. A 328 (2004), 127-143.

[22] Q. K. Song and Z. D. Wang, An analysis on existence and global exponential stability of periodic solutions for BAM neural networks with time-varying delays, Nonlinear Anal. Real World Appl. 8 (2007), no. 4, 1224-1234.

ZHENGQIU ZHANG

College of Mathematics

HunAN University

Changsha 410082, P. R. China

E-mail address: zhangzhengqiu@hnu.cn

Zheng Zhou

College of Mathematics

HunAN University

Changsha 410082, P. R. China

E-mail address: zhouzhenghnu@yahoo.com.cn 